\title{
Diagnostic accuracy of ultrasound superb microvascular imaging for breast tumor: a meta-analysis
}

\author{
Lin Zhong, Cong Wang
}

The First Affiliated Hospital of Dalian Medical University, Dalian City, Liaoning Province, China

\begin{abstract}
Aim: This meta-analysis aimed to identify the accuracy of ultrasound superb microvascular imaging (SMI) for the diagnosis of a breast tumor. Material and method: We searched PubMed, Web of Science, Google Scholar, Cochrane Library, EBSCO, and CBM databases from January 1st, 2013 until February 1st, 2020 without language restrictions. Meta-analysis was conducted using STATA version 14.0 and Meta-Disc version 1.4 software. We calculated the summary statistics for sensitivity (Sen), specificity (Spe), positive and negative likelihood ratio $\left(\mathrm{LR}^{+} / \mathrm{LR}^{-}\right)$, diagnostic odds ratio (DOR) and receiver operating characteristic (SROC) curve. Results: Fifteen studies that met all inclusion criteria were included in this meta-analysis. A total of 955 breast neoplasm patients and 1116 patients with benign breast tumors were assessed. All breast lesions were histologically confirmed after SMI. The pooled Sen was $0.81(95 \% \mathrm{CI}=0.78-0.83)$; the pooled Spe was $0.71(95 \% \mathrm{CI}=0.68-0.73) \mathrm{The}$ pooled $\mathrm{LR}^{+}$was $3.24(95 \% \mathrm{CI}=2.27-4.64)$; the pooled negative $\mathrm{LR}^{-}$was $0.25(95 \% \mathrm{CI}=0.18-0.34)$ The pooled DOR of SMI in the diagnosis of breast tumor was $46.97(95 \% \mathrm{CI}=16.72 \sim 131.97)$. The area under the SROC curve was $0.87(95 \% \mathrm{CI}=0.84-$ $0.90)$. We found no evidence for publication bias $(\mathrm{t}=-0.84, \mathrm{p}=0.42)$. Conclusion: Our meta-analysis indicates that SMI may have a high diagnostic accuracy in differential diagnosis between benign and malignant breast tumors. Thus, SMI may be a good tool for the diagnosis of breast tumors.
\end{abstract}

Keywords: Superb Microvascular Imaging; breast tumor; ultrasound; meta-analysis

\section{Introduction}

Breast cancer is a very common malignant disease in women worldwide [1]. It is predicted that more than one million women are diagnosed with breast cancer, and more than 400,000 will die from the disease every year [2]. Despite being the most common cancer, the 5 -year relative survival rate of breast carcinoma remains more than $80 \%$ when the disease is detected early [3]. To improve the survival, a number of screening methods to detect breast cancer have been studied, including magnetic resonance imaging (MRI), Doppler ultrasonog-

Received 19.02.2020 Accepted 22.04.2020

Med Ultrason

2020, Vol. 22, No 3, 313-318

Corresponding author: Cong Wang, MD

222 Zhongshan Road, Xigang District,

Dalian City, Liaoning Province, China

E-mail:wc027214@163.com

Phone: 18098877102 raphy (US) and computed tomography (CT) [4]. The most practical screening method in clinical diagnosis and treatment is US. The shape and distribution of neovascularization in tumors are related to the aggressive growth pattern of cancer cells, which is helpful to distinguish the nature of the tumor [5]. Color Doppler flow imaging (CDFI) is often used to show the blood flow inside the tumor, but CDFI can provide limited data in some low-velocity microvessels [6]. As a novel US technique, Superb Microvascular Imaging (SMI) can quickly, simply and noninvasively study the microvascular distribution in the tumor and evaluate the microvascular perfusion [7]. The SMI adopts a multidimensional filter to eliminate only the clutter and to preserve low-velocity flow signals, whereas conventional Doppler systems use a single-dimension filter and, accordingly, can exhibit a loss of low-velocity flow signals that overlap with clutter [8]. Previous studies have shown that SMI can detect the blood flow signals of neovascularization in tumors and is helpful for the differentiation between benign and ma- 
lignant breast tumors [9]. However, the results of these studies have been contradictory. Therefore, the present meta-analysis aimed at determining the accuracy of SMI in the differential diagnosis between benign and malignant breast tumors.

\section{Material and methods}

\section{Literature search}

We searched PubMed, Web of Science, Google Scholar, Cochrane Library, EBSCO and CBM databases from January 1st, 2013 until February 1st, 2020 without language restrictions. The following keywords and $\mathrm{MeSH}$ terms were used: ["breast cancer" or "breast neoplasms" or "breast tumor" or "mammary gland cancer" or "mammary gland neoplasms" or "mammary gland tumor"] and ["superb microvascular imaging"]. We also performed a manual search to find other potential articles.

\section{Selection criteria}

The following 4 criteria were required for each study: 1) the study design must be a clinical cohort study or diagnostic test; 2) the study must relate to the accuracy of SMI for the differential diagnosis of benign and malignant breast tumors; 3) all breast lesions were histologically confirmed after SMI; and 4) published data in the fourfold $(2 \times 2)$ tables must be sufficient. If the study did not meet all of these inclusion criteria, it was excluded. The most recent publication or the publication with the largest sample size was included when the authors published several studies using the same subjects.

\section{Data extraction}

Relevant data were systematically extracted from all included studies by two researchers using a standardized form. The researchers collected the following data: the first author's surname, publication year, language of publication, study design, sample size, number of lesions, source of the subjects, "gold standard," and diagnostic accuracy. The true positives (TP), true negatives (TN), false positives (FP), and false negatives (FN) in the fourfold $(2 \times 2)$ tables were also collected.

\section{Quality assessment}

Methodological quality was independently assessed by two researchers based on the quality assessment of studies of diagnostic accuracy studies (QUADAS) tool [10]. The QUADAS criteria included 14 assessment items. Each of these items was scored as "yes" (2), "no" (0), or "unclear"(1). The QUADAS score ranged from 0 to 28 , and a score $\geq 22$ indicated good quality.

\section{Statistical analysis}

The STATA version 14.0 (Stata Corp, College Station, TX, USA) and Meta-Disc version 1.4 (Universidad Complutense, Madrid, Spain) softwares were used for meta-analysis. We calculated the pooled summary statistics for sensitivity (Sen), specificity (Spe), positive and negative likelihood ratio (LR+/LR-), and diagnostic odds ratio (DOR) with their $95 \%$ confidence intervals (CIs). The summary receiver operating characteristic (SROC) curve and corresponding area under the curve (AUC) were obtained. The threshold effect was assessed using Spearman correlation coefficients. The Cochran's Q-statistic and I test were used to evaluate potential heterogeneity between studies. If significant heterogeneity was detected ( $Q$ test $\mathrm{p}<0.05$ or I test $>50 \%$ ), a random effects model or fixed effects model was used. We also performed subgroup and meta-regression analyses to investigate potential sources of heterogeneity. To evaluate the influence of single studies on the overall estimate, a sensitivity analysis was performed.

We conducted Begger's funnel plots and Egger's linear regression tests to investigate publication bias.

\section{Results}

\section{Characteristics of included studies}

Initially, the searched keywords identified 47 articles. We reviewed the titles and abstracts of all articles and excluded 21 articles; full texts and data integrity were also reviewed and 11 were further excluded. Finally, 15 studies that met all inclusion criteria were included in this meta-analysis [11-25]. Figure 1 showed the selection process of eligible articles. A total of 955 malignant breast lesions and 1116 benign breast tumors were assessed. We summarized the study characteristics and methodological quality in Table I. The QUADAS scores of all included studies were $\geq 22$.

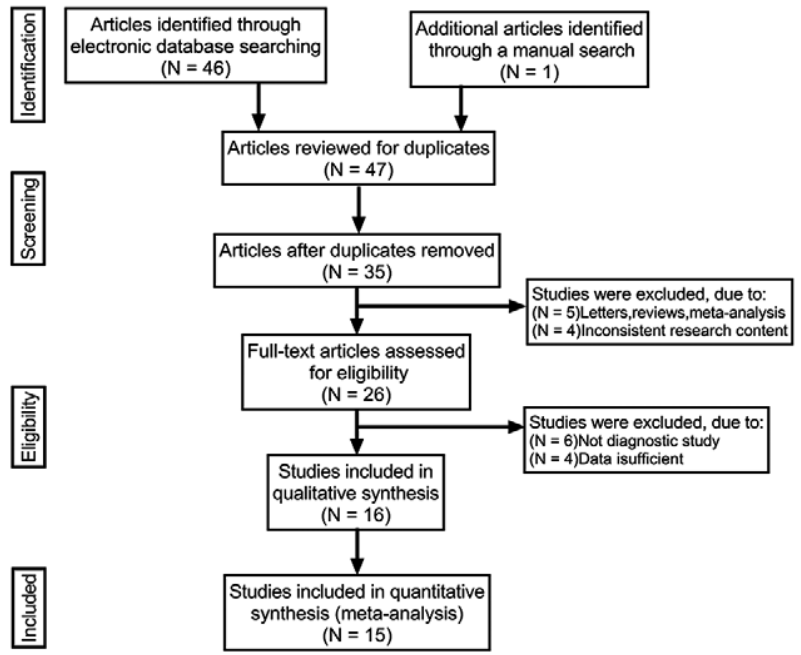

Fig 1. Flow chart of literature search and study selection. Fifteen studies were included in this meta-analysis. 
Table I. Baseline characteristics and methodological quality of all included studies

\begin{tabular}{|c|c|c|c|c|c|c|c|c|c|c|c|c|}
\hline \multirow[t]{2}{*}{ First author } & \multirow[t]{2}{*}{ Year } & \multirow{2}{*}{$\begin{array}{l}\text { Coun- } \\
\text { try }\end{array}$} & \multirow{2}{*}{$\begin{array}{l}\text { Lan- } \\
\text { guage }\end{array}$} & \multirow{2}{*}{$\begin{array}{l}\text { Sample } \\
\text { size }\end{array}$} & \multirow{2}{*}{$\begin{array}{l}\text { Age } \\
\text { (Years) }\end{array}$} & \multirow{2}{*}{$\begin{array}{l}\text { Instrument } \\
\text { (Toshiba) }\end{array}$} & \multirow[t]{2}{*}{ Diagnostic basis } & \multicolumn{4}{|c|}{ SMI $2 \times 2$ table } & \multirow{2}{*}{$\begin{array}{l}\text { QUADAS } \\
\text { score }\end{array}$} \\
\hline & & & & & & & & $\mathrm{TP}$ & FP & $\mathrm{FN}$ & $\mathrm{TN}$ & \\
\hline Wang [11] & 2020 & China & Chinese & 160 & $54.1 \pm 11.2$ & Aplio 500 & Adler classification & 97 & 3 & 27 & 31 & 23 \\
\hline Zhang [12] & 2019 & China & Chinese & 252 & $46.7 \pm 12.5$ & Aplio 500 & Morphology type & 105 & 10 & 13 & 124 & 24 \\
\hline $\mathrm{Ni}$ [13] & 2019 & China & Chinese & 123 & $55.6 \pm 9.0$ & Aplio & Adler classification & 51 & 20 & 2 & 50 & 24 \\
\hline Xiao & 2019 & China & & 105 & $47.2 \pm 10.5$ & Aplio 500 & Adler classification & 34 & 13 & 7 & 51 & 22 \\
\hline Che [15] & 2018 & China & Chinese & 88 & $48.5 \pm 10.5$ & Aplio 400 & Adler classification & 30 & 26 & 10 & 22 & $2 J$ \\
\hline Yao [16] & 2017 & China & Chinese & 102 & $44.5 \pm 11.9$ & Aplio 500 & Adler classification & 41 & 4 & 26 & 31 & 22 \\
\hline Ming [17] & 2019 & China & English & 177 & $43.9 \pm 14.1$ & Aplio 400 & Morphology type & 42 & 6 & 21 & 93 & 24 \\
\hline Zhu [18] & 2019 & China & English & 116 & $47.1 \pm 9.3$ & Aplio 500 & Adler classification & 43 & 56 & 3 & 30 & 23 \\
\hline Zhang [19] & 2020 & China & English & 236 & $49.4 \pm 12.5$ & Aplio 500 & Vascularity index & 44 & 39 & 29 & 76 & 25 \\
\hline $\mathrm{Ah}[20]$ & 2019 & Korea & English & 98 & $45.6 \pm 9.7$ & Aplio 500 & Synthesis index & 45 & 14 & 9 & 43 & 22 \\
\hline Suleyman [21] & 2019 & Korea & & 54 & $48.5 \pm 11.2$ & 00 & grading & 46 & 6 & 2 & 33 & 23 \\
\hline Park [22] & 2018 & Korea & & 191 & $47(20-83)$ & & & 47 & 34 & 16 & 65 & 24 \\
\hline Zhao [23] & 2016 & China & English & 135 & $46.9 \pm 10.4$ & Aplio 500 & Vascular grading & 48 & 18 & 8 & 76 & 24 \\
\hline $\mathrm{Ma}[24]$ & 2015 & China & English & 123 & $45.1(15-69)$ & Aplio 400 & Adler classification & 49 & 27 & 6 & 39 & 23 \\
\hline Xiao [25] & 2016 & China & English & 132 & $44.1(16-78)$ & Aplio 400 & Adler classification & 50 & 48 & 4 & 26 & 25 \\
\hline
\end{tabular}

$\mathrm{TP}$, true positive; TN, true negative; FP, false positive; FN, false negative; QUADAS, the quality assessment of studies of diagnostic accuracy studies

\section{Quantitative data synthesis}

Meta-analysis findings on the accuracy of SMI for differential diagnosis between benign and malignant breast tumors were showed in Table II. The random effects model was used due to obvious heterogeneity among the studies. Diagnostic accuracy of SMI was measured as pooled Sen, Spe, $\mathrm{LR}^{+}, \mathrm{LR}^{-}$and DOR (fig 2). Our meta-analysis revealed that the pooled Sen was $0.81(95 \% \mathrm{CI}=0.78$ $0.83)$; the pooled Spe was $0.71(95 \% \mathrm{CI}=0.68-0.73)$. There was no significant correlation $(\mathrm{r}=243, \mathrm{p}=0.610)$ between sensitivity and specificity, which indicated that there is no threshold effect. In addition, we observed that the pooled $\mathrm{LR}^{+}$and $\mathrm{LR}^{-}$were $3.24(95 \% \mathrm{CI}=2.27-4.64)$ and $0.25(95 \% \mathrm{CI}=0.18-0.34)$, respectively. The pooled DOR of SMI in the diagnosis of breast tumor was 15.16 (95\% CI=8.24-27.87) (fig 3). The results were plotted as a symmetrical SROC curve (fig 4) and the corresponding AUC was $0.87(95 \% \mathrm{CI}=0.84-0.90)$. Subgroup and meta-regression analyses were conducted based on language, instrument type, and diagnostic basis to investigate potential sources of heterogeneity. In the subgroup analyses, the results revealed that SMI exhibited a high diagnostic performance in different subgroups (Table II). Meta-regression analysis results confirmed that no factor could explain the potential sources of heterogeneity (Table III). We found no evidence of obvious asymmetry in the Begger's funnel plots (fig 5). Egger's test also did not display strong statistical evidence for publication bias $(\mathrm{t}=-0.84, \mathrm{p}=0.42)$.
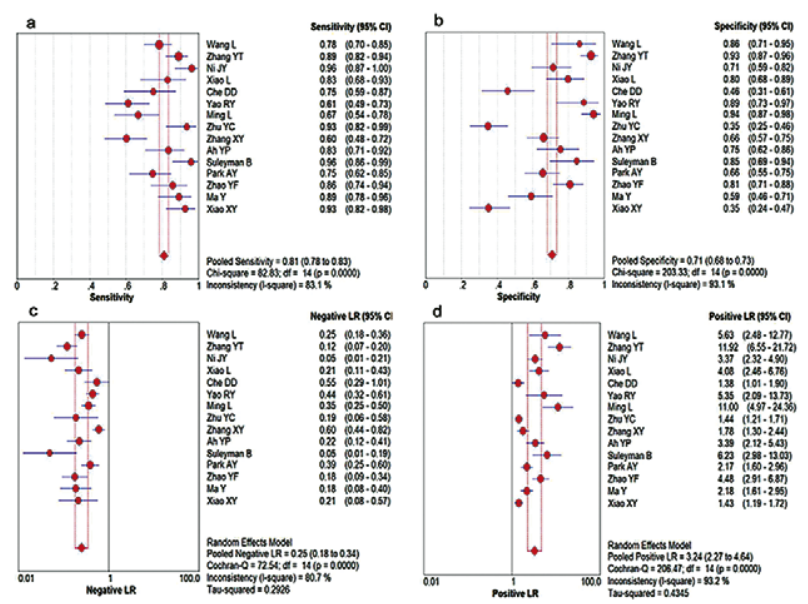

Fig 2. Forest plots for the accuracy of SMI for the diagnosis of breast tumors. a Sensitivity. b Specificity. c Positive likelihood ratio. $\mathrm{d}$ Negative likelihood ratio.

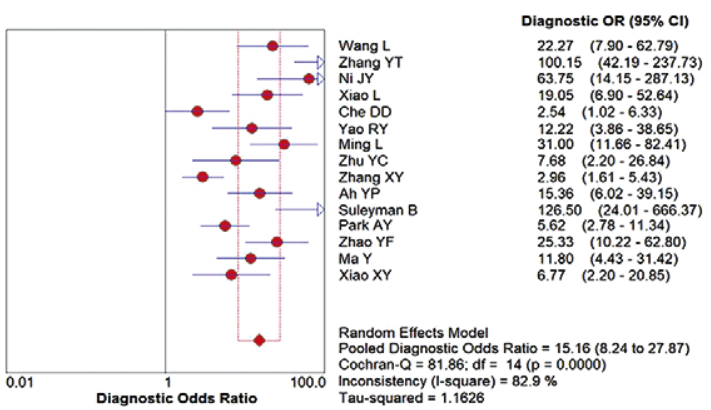

Fig 3. Forest plot of DOR of SMI for the diagnosis of breast tumors. DOR diagnostic odds ratio 


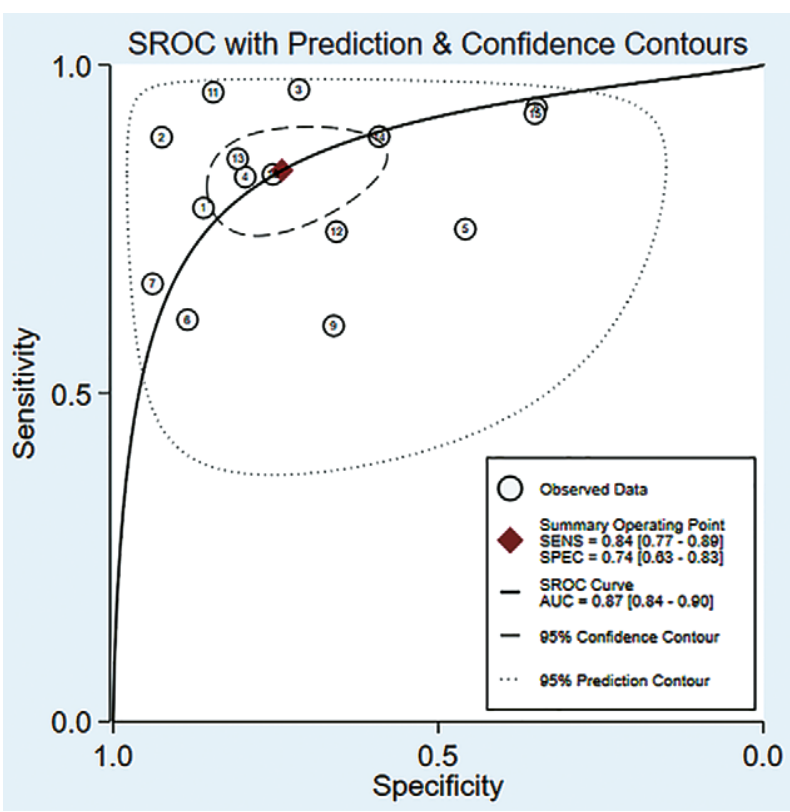

Fig 4. SROC curve for the accuracy of SMI in the diagnosis of breast. SROC summary receiver operator characteristic, AUC area under curve.

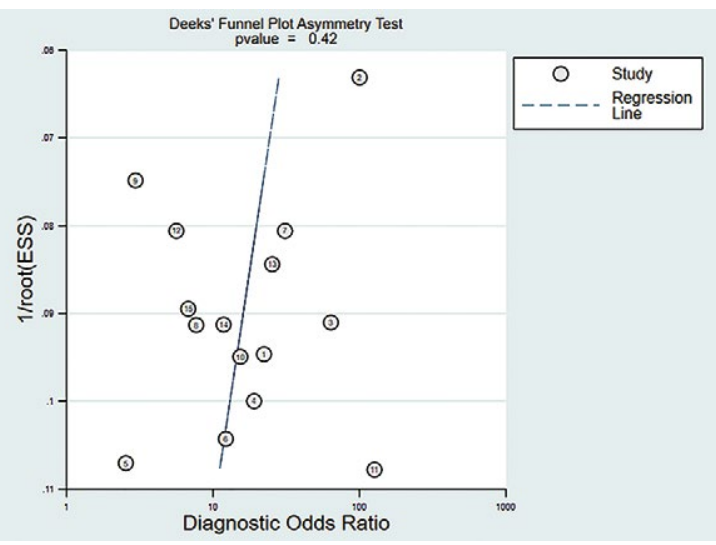

Fig 5. Begger's funnel plot of publication bias on the pooled OR. No publication bias was detected

\section{Discussion}

With the wide use of high-resolution ultrasound diagnostic instruments, the detection rate of breast nodules has increased significantly [7]. The growth of tumor depends on the complex blood vessels inside the tumor.

Table II. Meta-analysis of the accuracy of SMI for the diagnosis of breast tumors

\begin{tabular}{|c|c|c|c|c|c|c|}
\hline Subgroup & $\begin{array}{l}\text { Studies } \\
\text { (n) }\end{array}$ & $\begin{array}{l}\text { Sen } \\
(95 \% \mathrm{CI})\end{array}$ & $\begin{array}{l}\text { Spe } \\
(95 \% \mathrm{CI})\end{array}$ & $\begin{array}{l}\mathrm{LR}^{+} \\
(95 \% \mathrm{CI})\end{array}$ & $\begin{array}{l}\text { LR }^{-} \\
(95 \% \mathrm{CI})\end{array}$ & $\begin{array}{l}\text { DOR } \\
(95 \% C I)\end{array}$ \\
\hline Overall & 15 & $0.81[0.78-0.83]$ & $0.71[0.68-0.73]$ & $3.24[2.27-4.64]$ & $0.25[0.18-0.34]$ & $15.16[8.24-27.87]$ \\
\hline \multicolumn{7}{|l|}{ Language } \\
\hline Chinese & 6 & $0.81[0.77-0.84]$ & $0.80[0.75-0.84]$ & $4.24[2.02-8.90]$ & $0.23[0.13-0.42]$ & $20.48[6.49-64.57]$ \\
\hline English & 9 & $0.81[0.77-0.84]$ & $0.66[0.62-0.69]$ & $2.72[1.87-3.94]$ & $0.26[0.17-0.39]$ & $12.24[6.20-24.18]$ \\
\hline \multicolumn{7}{|l|}{ Ultrasound machine } \\
\hline Toshiba Aplio 500 & 11 & $0.83[0.80-0.85]$ & $0.73[0.70-0.76]$ & $3.60[2.23-5.81]$ & $0.22[0.15-0.33]$ & $18.54[9.06-37.93]$ \\
\hline Toshiba Aplio 400 & 4 & $0.76[0.70-0.81]$ & $0.66[0.61-0.71]$ & $2.47[1.40-4.35]$ & $0.34[0.20-0.58]$ & $8.94[3.00-26.65]$ \\
\hline \multicolumn{7}{|l|}{ Diagnostic basis } \\
\hline Vascularity index & 1 & $0.60[0.48-0.72]$ & $0.66[0.57-0.75]$ & $1.78[1.30-2.44]$ & $0.60[0.44-0.82]$ & $2.96[1.61-5.43]$ \\
\hline Synthesis index & 2 & $0.79[0.70-0.86]$ & $0.69[0.61-0.76]$ & $2.62[1.69-4.07]$ & $0.30[0.18-0.53]$ & $8.84[3.31-23.58]$ \\
\hline Vascular grading & 2 & $0.90[0.83-0.95]$ & $0.82[0.74-0.88]$ & $4.87[3.36-7.05]$ & $0.11[0.03-0.37]$ & $48.42[10.30-227.71]$ \\
\hline Morphology type & 2 & $0.70[0.61-0.78]$ & $0.81[0.75-0.87]$ & $11.58[7.17-18.69]$ & $0.21[0.07-0.65]$ & $57.02[18.07-179.94]$ \\
\hline Adler classification & 8 & $0.82[0.79-0.86]$ & $0.58[0.54-0.63]$ & $2.41[1.66-3.49]$ & $0.25[0.17-0.39]$ & $11.52[6.05-21.93]$ \\
\hline
\end{tabular}

95\%CI, 95\% confidence interval; LR, likelihood ratio; DOR, diagnostic odds ratio; Sen, Sensitivity; Spe, Specificity

Table III. Meta-regression analyses of potential source of heterogeneity

\begin{tabular}{lllllll}
\hline Heterogeneity factors & Coefficient & SE & p value & RDOR & & \multicolumn{2}{c}{ 95\% CI } \\
\cline { 5 - 7 } & & & & & UL & LL \\
\hline Publication year & -0.002 & 0.3136 & 0.9962 & 1.00 & 0.49 & 2.03 \\
Language & 0.179 & 1.0684 & 0.8704 & 0.84 & 0.07 & 9.37 \\
Instrument & 0.715 & 0.9390 & 0.4641 & 0.49 & 0.06 & 4.09 \\
Diagnostic basis & 0.084 & 0.4321 & 0.8503 & 0.92 & 0.35 & 2.44 \\
\hline
\end{tabular}

SE, standard error; RDOR, relative diagnostic odds ratio; 95\%CI, $95 \%$ confidence interval; UL, upper limit; LL, lower limit 
After the proangiogenic and angiogenic stages, the tumor gradually infiltrates and expands to the peripheral tissue. When the tumor is in the proangiogenesis (diameter $<2$ $\mathrm{mm}$ ), the invasion range is limited, but when the tumor enters the angiogenic stage (diameter $>2 \mathrm{~mm}$ ), the blood vessels will extend more branches, form a large number of capillaries, infiltrate to the surrounding area and absorb a large amount of nutrients for the survival of the tumor, and at the same time, tumor invasion and metastasis will occur [26]. The vascular morphology and distribution of breast masses are closely related to the nature of the tumor, which can be used as an important supplementary sign in their differential diagnosis [17].

SMI can clearly and completely show the shape and distribution of the vascular network of breast mass without injection of a contrast agent. By observing the number and distribution of blood vessels of breast cancer, SMI can help to judge the nature of the tumor [19]. According to Adler's method, the vascularity was subjectively determined to be absent (grade 0), minimal (grade 1), moderate (grade 2) or marked (grade 3). The blood flow classification of benign tumors is mostly $0-1$, while that of malignant tumors is mostly 2-3. Compared with benign tumors, the blood supply of malignant tumors is more abundant and the detection rate of blood flow is higher [24]. SMI can display microvascular architecture patterns of breast tumors. Of that benign masses tend to display the non-vascular pattern, a linear or curvilinear pattern and a treelike pattern, while malignant masses tend to display the root hair-like pattern and the crab claw-like pattern [12]. Smart 3-D SMI can quantitatively assess tumor vascularity via measuring vascularity index (VI) on 2-D SMI images obtained with the qualitative guidance of 3-D SMI images, which is significantly higher in malignant lesions than that in benign ones and may potentially serve as a noninvasive tool to accurately characterize benign versus malignant breast lesions [20]. Present studies have revealed that SMI is more accurate in the detection and characterization of breast lesions comparable with mammography X-ray and conventional CDFI scanning [15]. Nevertheless, each imaging diagnostic examination has its own advantages and disadvantages and only one imaging examination is generally not enough to make an accurate diagnosis of the disease. Therefore, SMI will not replace but can only compliment conventional ultrasound. Although considered as a potentially useful imaging tool, SMI is still not widely used in clinical practice and few reports have discussed its use in the evaluation of breast tumors. This controversy could be caused by several factors, including the differences in study designs, sample size, number of lesions, diagnostic criteria, statistical methods, etc.
In the present meta-analysis, we systematically evaluated the technical performance and accuracy of SMI for differential diagnosis of benign and malignant breast tumors. The 15 independent studies included assessed 955 malignant breast lesions and 1116 benign breast tumors. The pooled Sen, Spe and DOR of SMI in the diagnosis of breast cancer were $0.81,0.71$ and 46.97 , respective1y. These results were consistent with the potentially high diagnostic accuracy of SMI for breast cancer, suggesting that SMI may be a good tool for the differential diagnosis of benign and malignant breast tumors and could predict the prognosis of breast cancer patients. The threshold effect is usually interpreted as a sudden and radical change in a phenomenon that often occurs after surpassing a quantitative limit. Our findings showed no significant relationship between Sen and Spe within the studies, providing no evidence of a threshold effect. Because heterogeneity existed in the individual studies, subgroup analyses were conducted. SMI exhibited a high diagnostic performance in different subgroups for the diagnosis of breast cancer, suggesting that differences in language, diagnostic basis and instrument type did not directly influence the diagnostic accuracy of SMI. Furthermore, our results found no direct evidence of publication bias. Collectively, our findings strongly suggest that SMI is a highly accurate and non-invasive tool for the qualitative diagnosis of breast cancer, consistent with previous studies.

Despite the demonstrated diagnostic accuracy of SMI for breast cancer, our study has certain limitations. First, owing to the relatively small sample sizes and low level of quality of the included studies, there were insufficient data to assess the accuracy of SMI. Moreover, the retrospective nature of a meta-analysis can lead to subject selection bias. Importantly, the majority of included studies originated from China, which may adversely affect the reliability and validity of our results.

In conclusion, our meta-analysis suggests that SMI may have high diagnostic accuracy in the differential diagnosis of benign and malignant breast tumors. Thus, SMI may be a good tool to diagnose breast cancer. However, due to the limitations, further detailed studies are required to confirm the present findings.

Acknowledgments: we would like to thank all our colleagues working in the Ultrasound Department of the First Affiliated Hospital to Dalian Medical University.

\section{Conflicts of interest: none}

\section{References}

1. Akram M, Iqbal M, Daniyal M, Khan AU. Awareness and current knowledge of breast cancer. Biol Res 2017;50:33. 
2. Ogunkorode A, Holtslander L, Anonson J, Maree J. Promoting Early Detection of Breast Cancer and Care Strategies for Nigeria. Afr J Reprod Health 2017;21:18-25.

3. Brenner H, Gundos A, Arndt U. Recent major progress in long-term cancer patient survival disclosed by modeled period analysis. J Clin Oncol 2007;25:3274-3280.

4. Takkar N, Kochhar S, Garg P, Pandey AK, Dalal UR, Handa U. Screening methods (clinical breast examination and mammography) to detect breast cancer in women aged 4049 years. J Midlife Health 2017;8:2-10.

5. Maniotis AJ, Folberg R, Hess A, et al. Vascular channel formation by human melanoma cells in vivo and in vitro: vasculogenic mimicry. Am J Pathol 1999;155:739-752.

6. Wang XN, Zhao Q, Li DJ, et al. Quantitative evaluation of primary retinitis pigmentosa patients using colour Doppler flow imaging and optical coherence tomography angiography. Acta Ophthalmol 2019;97:e993-e997.

7. Hata T, Koyanagi A, Yamanishi T, Bouno S, Takayoshi R, Miyake T. Superb microvascular imaging with Doppler luminance using an $18-\mathrm{MHz}$ probe to visualize fetal intraabdominal blood vessels and organ microvasculature. J Perinat Med 2020;48:184-188.

8. Jiang ZZ, Huang YH, Shen HL, Liu XT. Clinical Applications of Superb Microvascular Imaging in the Liver, Breast, Thyroid, Skeletal Muscle, and Carotid Plaques. J Ultrasound Med 2019;38:2811-2820.

9. ParkAY,SeoBK.Up-to-dateDopplertechniques forbreasttumor vascularity: superb microvascular imaging and contrastenhanced ultrasound. Ultrasonography 2018;37:98-106.

10. Whiting PF, Weswood ME, Rutjes AW, Reitsma JB, Bossuyt PN, Kleijnen J. Evaluation of QUADAS, a tool for the quality assessment of diagnostic accuracy studies. BMC Med Res Methodol 2006;6:9.

11. Wang L, Deng KX, Sui XF, et al. Screening and diagnosis value of acoustic radiation force impulse imaging quantification in combination with superb microvascular imaging in early breast cancer. Chinese General Practice 2020. [Epub ahead of print].

12. Yanting Z, Li M, Xinhui M, Fuxia W, Wen W. Value Study of Superb Microvascular Imaging on BI-RADS Category 3 and 4 Breast Masses, Chinese Journal of Ultrasound in Medicine 2019;35:975-978.

13. Ni Jing Yuan. Application value of shear wave elastography combined with microvascular imaging in differential diagnosis of benign and malignant breast masses. 2019[D]: Jilin University.

14. Xiao Lu. The preliminary analysis and diagnostic value of microvasculature of breast tumor by ultra micro angiography. 2019[D]: Henan University of science and technology.
15. Che Dandan. Diagnosis of breast masses by ultra micro angiography combined with molybdenum target X-ray. 2018[D]: Jinzhou Medical University.

16. Yao Ruiyan, Wu Liyuan, Xu Xiaohong, Diagnostic value of superb microvascular imaging versus color Doppler flow imaging for breast nodules. Journal of Guangdong Medical University 2017;35:173-176.

17. Liang M, Ou B, Wu J, et al. Combined use of strain elastography and superb microvascular imaging with grayscale ultrasound according to the BI-RADS classification for differentiating benign from malignant solid breast masses. Clin Hemorheol Microcirc 2019 Nov 1. doi:10.3233/CH190693.

18. Zhu YC, Zu DM, Zhang Y, et al. A comparative study on superb microvascular imaging and conventional ultrasonography in differentiating BI-RADS 4 breast lesions. Oncol Lett 2019;18:3202-3210.

19. Zhang XY, Zhang L, Li N, et al. Vascular index measured by smart 3-D superb microvascular imaging can help to differentiate malignant and benign breast lesion. Cancer Manag Res 2019;11:5481-5487.

20. Park AY, Kwon M, Woo OH, et al. A Prospective Study on the Value of Ultrasound Microflow Assessment to Distinguish Malignant from Benign Solid Breast Masses: Association between Ultrasound Parameters and Histologic Microvessel Densities. Korean J Radiol 2019;20:759-772.

21. Bakdik S, Arslan S, Oncu F, et al. Effectiveness of Superb Microvascular Imaging for the differentiation of intraductal breast lesions. Med Ultrason 2018;20:306-312.

22. Park AY, Seo BK, Woo OH, et al. The utility of ultrasound superb microvascular imaging for evaluation of breast tumour vascularity: comparison with colour and power Doppler imaging regarding diagnostic performance. Clin Radiol 2018;73:304-311.

23. Yongfeng Z, Ping Z, Wengang L, Yang S, Shuangming T. Application of a Novel Microvascular Imaging Technique in Breast Lesion Evaluation. Ultrasound Med Biol 2016;42:2097-2105.

24. Ma Y, Li G, Li J, Ren WD. The Diagnostic Value of Superb Microvascular Imaging (SMI) in Detecting Blood Flow Signals of Breast Lesions: A Preliminary Study Comparing SMI to Color Doppler Flow Imaging. Medicine (Baltimore) 2015;94:e1502.

25. Xiao XY, Chen X, Guan XF, Wu H, Qin W, Luo BM. Superb microvascular imaging in diagnosis of breast lesions: a comparative study with contrast-enhanced ultrasonographic microvascular imaging. Br J Radiol 2016;89:20160546.

26. Carmeliet P, Jain RK. Angiogenesis in cancer and other diseases. Nature 2000;407:249-257. 